\title{
COMMUTING ANALYTIC FUNCTIONS
}

BY

CARL C. COWEN ${ }^{1}$

\begin{abstract}
Let $f$ and $g$ (not conformal automorphisms of the unit disk) be analytic mappings of the unit disk into itself. We say $f$ and $g$ commute if $f \circ g=g \circ f$. This paper characterizes those functions $g$ that commute with a given function $f$. Several corollaries of this characterization give qualitative information about $g$ given similar information about $f$, and examples are given in each case to show the limitations of the conclusions. Some of the qualitative properties considered are univalence, fixed point sets, and whether two such $g$ must commute with each other.
\end{abstract}

1. Introduction. Let $f$ and $g$ be nonconstant analytic functions mapping the unit disk $D$ into itself. We say $f$ and $g$ commute if $f \circ g=g \circ f$. This paper investigates the relationship between commuting analytic functions. (To avoid trivialities, we assume neither $f$ nor $g$ is a conformal automorphism of $D$.)

Obviously, if $f=h \circ h$ and $g=h \circ h \circ h$ for some function $h$, then $f$ and $g$ commute. The functions $f_{n}=f \circ f_{n-1}$ defined inductively for the natural numbers (where $f_{1}=f$ ) will be called the natural iterates of $f$. We will formulate $(\$ 2)$ a generalization of the natural iterates of $f$, the pseudo-iteration semigroup of $f$, and prove a theorem which, together with results of $\$ 2$, characterizes commuting functions:

THEOREM 3.1. Let $f$ and $g$ be nonconstant analytic functions mapping the unit disk into itself (not conformal automorphisms of $D$ ). If $f \circ g=g \circ f$, then $f$ and $g$ are in the pseudo-iteration semigroup of the same function.

It is seldom possible to find explicitly the pseudo-iteration semigroup of a function, but this theorem is strong enough to deduce qualitative properties of functions commuting with a function from similar qualitative information about the function. $\$ 4$ is devoted to exploring these relationships, including giving several examples that limit the scope of the consequences. The questions investigated include whether $f$ univalent implies all functions commuting with $f$ are, whether all functions commuting with $f$ have the same fixed point set, and whether two functions commuting with $f$ must commute with each other.

Received by the editors June 6, 1982 and, in revised form, June 15, 1983. These results were presented to the American Mathematical Society at the winter meeting in Cincinnati, Ohio, January 14, 1982.

1980 Mathematics Subject Classification. Primary 30D05; Secondary 39B10.

Key words and phrases. Functional equation, commuting functions.

${ }^{1}$ Supported in part by National Science Foundation Grant MCS-7902018. 
The problem of characterizing functions commuting with a given function has been studied by several authors in various contexts. Julia [11], Fatou [7], Ritt [13, 14, 15] and Jacobsthal [10] have studied the problem for rational functions. Baker studied the problem for entire functions [1] and for functions of the form $f(z)=z$ $+\cdots$ [2]. Hadamard [9] reports on work on functions of the form $f(z)=c z+\cdots$. Because their special assumptions were different from ours $(f(D) \subset D)$, the results of their work suggest questions for study but, in most cases, are not directly applicable. Indeed, these authors might have considered some of these results surprising. Hadamard wrote [9, p. 73], “We also see Lüntz was right in supposing that two functions reversible to the same third one are reversible to each other," but Example 4.4 shows that for analytic functions mapping the disk into itself, this need not be true.

The works that study this problem specifically for the disk are those of Pranger [12], Shields [16], and Behan [3]. Pranger considers commutation with functions mapping $D$ into $D$ that have a fixed point in $D$ and are locally univalent. Theorem 3.1, for this case, is the same as Pranger's Theorem 1 [12, p. 202] and the proofs are the same. Pranger also characterizes [12, Theorem 3, p. 202] the semigroups that can arise as semigroups of functions commuting with a function of the given form.

As is always the case in investigating questions of this type, the nature of the fixed points of $f$ is important. We will say a point $b$ in $\bar{D}$ is a fixed point of $f$ if $\lim _{r \rightarrow 1^{-}} f(r b)=b$. If $b$ is a fixed point of $f, \lim _{r \rightarrow 1^{-}} f^{\prime}(r b)$ exists, and we write $f^{\prime}(b)$ for this limit. (If $|b|<1$, the Schwarz lemma implies $\left|f^{\prime}(b)\right|<1$, and if $|b|=1, f^{\prime}(b)$ must be real and positive (or infinite).) A theorem of Denjoy and Wolff guarantees the existence of a distinguished fixed point:

THEOREM D-W [6, 18]. If $f$ is an analytic mapping of $D$ into $D$, not a conformal automorphism of $D$, there is a unique fixed point $a$ in $\bar{D}$ of $f$ such that $\left|f^{\prime}(a)\right| \leqslant 1$.

The distinguished fixed point $a$ mentioned in the theorem above will be called the Denjoy-Wolff point of $f$. Shields [16] (for functions continuous on the closed disk) and Behan [3] (generally) showed that if $f$ and $g$ commute then the Denjoy-Wolff points of $f$ and $g$ are the same. This fact is the beginning of our work.

I would like to thank the referee and Larry Brown for several helpful suggestions.

2. The pseudo-iteration semigroup. Before defining the pseudo-iteration semigroup of a function, we note some necessary background. Throughout this section we suppose $f$ is a nonconstant analytic mapping of $D$ into itself, not an automorphism of $D$, with Denjoy-Wolff point $a$.

DeFinition [4, p. 76]. If $f$ maps a domain $\Delta$ into itself, we say $V$ is a fundamental set for $F$ on $\Delta$ if $V$ is an open, connected, simply connected subset of $\Delta$ such that $F(V) \subset V$ and for every compact set $K$ in $\Delta$, there is a positive integer $n$ such that $F_{n}(K) \subset V$.

A fundamental set is large enough to understand the iterates of $F$ on all of $\Delta$; this concept provides the uniqueness in the following structure theorem which is crucial to the later results. 
TheOREM A [4, TheOREM 3.2, p. 78]. Suppose $f$, as above, has $f^{\prime}(a) \neq 0$. Then there is a fundamental set $V$ for $f$ on $D$, a domain $\Omega$, either the complex plane or the unit disk, a linear fractional transformation $\Phi$ mapping $\Omega$ onto $\Omega$ and an analytic mapping $\sigma$ of $D$ into $\Omega$ such that $f$ and $\sigma$ are univalent on $V, \sigma(V)$ is a fundamental set for $\Phi$ on $\Omega$ and $\Phi \circ \sigma=\sigma \circ f$. Moreover, $\Omega$ is unique and $\Phi$ and $\sigma$ are essentially unique: if $\tilde{\Phi}, \tilde{\sigma}$, $\tilde{V}$ also satisfy the above, there is a linear fractional transformation $\varphi$ mapping $\Omega$ onto $\Omega$ so that $\tilde{\Phi}=\varphi \circ \Phi \circ \varphi^{-1}$ and $\tilde{\sigma}=\varphi \circ \sigma$.

In case $f^{\prime}(a)=0$ we may assume, without loss of generality, that $a=0$.

TheOREM B [17, pp. 124-127] (cf. [5]). Suppose $f(z)=\alpha z^{k}+\cdots$, where $\alpha \neq 0$ and $k \geqslant 2$, is as above. There is a function $\sigma$ analytic and univalent in a neighborhood of 0 such that $\sigma(f(z))=[\sigma(z)]^{k}$. Moreover, if $\tilde{\sigma}$ is another such function, $\tilde{\sigma}=\lambda \sigma$, where $\lambda^{k-1}=1$.

We are now ready to define "pseudo-iteration semigroup". The defintion is in two cases: $f^{\prime}(a)=0$ and $f^{\prime}(a) \neq 0$.

Case 1: $f^{\prime}(a)=0$. Without loss of generality, $f(z)=\alpha z^{k}+\cdots$ where $\alpha \neq 0$ and $k \geqslant 2$. Let $\sigma$ be as in Theorem B. We say $g$, an analytic mapping of $D$ into $D$, is in the pseudo-iteration semigroup of $f$ if there is a positive integer $m$ and a number $\lambda$ with $\lambda^{k-1}=1$ such that $\sigma(g(z))=\lambda[\sigma(z)]^{m}$ in some neighborhood of 0 .

Case 2: $f^{\prime}(a) \neq 0$. Let $\Omega, V, \sigma$ and $\Phi$ be as in Theorem A. We say $g$, an analytic mapping of $D$ into $D$, is in the pseudo-iteration semigroup of $f$ if there is a linear fractional transformation $\psi$ that commutes with $\Phi$ such that $\sigma(g(z))=\psi(\sigma(z))$.

It is easy to verify from the uniqueness statements of Theorems A and B that the pseudo-iteration semigroup does not depend on the choice of $\sigma$. The pseudo-iteration semigroup is a semigroup under composition: if $g$ and $h$ are in the pseudo-iteration semigroup of $f$, so is $g \circ h$. Moreover, the pseudo-iteration semigroup of $f$ contains all the natural iterates of $f: \sigma \circ f_{n}=\sigma^{k^{n}}$ in Case 1 and $\sigma \circ f_{n}=\Phi_{n} \circ \sigma$ in Case 2.

In Case 1, since $\sigma$ is univalent in some neighborhood of 0 and $g(0)=0$, there is a small disk with center zero on which $g(z)=\sigma^{-1}\left(\lambda[\sigma(z)]^{m}\right)$, where $\sigma^{-1}$ denotes the inverse of the restriction of $\sigma$. The local character of the definition in Case 1 is unavoidable because, first, there may be no solutions of Böttcher's functional equation $\sigma \circ f=\sigma^{k}$ analytic on $D$ and, second, the solutions of Böttcher's equation analytic on large subdisks of $D$ may not be univalent near zero [5].

In Case 2, every $g$ in the pseudo-iteration semigroup of $f$ is a branch of $\sigma^{-1} \circ \psi \circ \sigma$ but it is less convenient, in the definition, to focus on the difficulty with branches since $\sigma$ is defined on all of $D$. Since $\Phi(\sigma(V)) \subset \sigma(V)$, we have $f(z)=\sigma^{-1}(\Phi(\sigma(z)))$, where $\sigma^{-1}$ denotes the inverse of the restriction of $\sigma$ to $V$. The major technical difficulty in this study is the necessity of dealing with the branch of $\sigma^{-1}$ mapping $\sigma(V)$ into $V$. The following propositions begin to explain the relation between pseudo-iteration semigroups and commutation.

Proposition 2.1. If $f$ is as above and $g$ is in the pseudo-iteration semigroup of $f$, then there is an integer $n$ such that $f_{n} \circ g$ and $f$ commute. 
Proof. Let $a$ be the Denjoy-Wolff point of $f$.

Case 1: $f^{\prime}(a)=0$. Without loss of generality, $f(z)=\alpha z^{k}+\cdots$, where $\alpha \neq 0$ and $k \geqslant 2$. Let $\sigma$ be as in Theorem B. From the preceding paragraphs, we have that on some disk with center zero $f(z)=\sigma^{-1}\left([\sigma(z)]^{k}\right)$ and $g(z)=\sigma^{-1}\left(\lambda[\sigma(z)]^{m}\right)$, where $\lambda^{k-1}=1$ and $m$ is a positive integer. Now, for $z$ near zero,

$$
\begin{aligned}
f(g(z)) & =\sigma^{-1}\left(\left[\sigma\left(\sigma^{-1}\left(\lambda[\sigma(z)]^{m}\right)\right)\right]^{k}\right) \\
& =\sigma^{-1}\left(\lambda^{k}[\sigma(z)]^{k m}\right)=\sigma^{-1}\left(\lambda[\sigma(z)]^{k m}\right) \\
& =\sigma^{-1}\left(\lambda\left[\sigma\left(\sigma^{-1}\left([\sigma(z)]^{k}\right)\right)\right]^{m}\right)=g(f(z)) .
\end{aligned}
$$

So $f$ and $g$ commute in a neighborhood of zero, hence on $D$, so we may take $n=0$ in the conclusion.

Case 2: $f^{\prime}(a) \neq 0$. Let $\Phi, \sigma, V$ and $\Omega$ be as in Theorem A. Let $K=\{z:|z| \leqslant 1 / 2\}$. Since $V$ is a fundamental set for $f$, there is an integer $n$ large enough that $f_{n+1}(g(K)) \subset V$ and $f_{n}(g(f(K))) \subset V$. Since $g$ is in the pseudo-iteration semigroup of $f, \sigma \circ g=\psi \circ \sigma$, where $\psi$ is a linear fractional transformation commuting with $\Phi$, so

$$
\sigma\left(f_{n}(g(f(z)))\right)=\Phi_{n}(\psi(\Phi(\sigma(z))))=\Phi_{n+1}(\psi(\sigma(z)))=\sigma\left(f_{n+1}(g(z))\right) .
$$

Now $\sigma$ is univalent on $V$, so for $z$ in $K$, this means $f_{n}(g(f(z)))=f_{n+1}(g(z))$. Since $K$ has nonempty interior, this holds for all $z$ in $D$ and $f$ commutes with $f_{n} \circ g$.

In general, the conclusion of Proposition 2.1 cannot be strengthened to read " $f$ commutes with $g$ ", although the proof for the case $f^{\prime}(a)=0$ gives this conclusion. For example, if $f(z)=\frac{1}{2}\left(1+z^{2}\right)$ and $g(z)=-\frac{1}{2}\left(1+z^{2}\right)$, then $f \circ g \neq g \circ f$, but since $f(z)=f(-z)$, it follows [4] that $\sigma(z)=\sigma(-z)$ so that $\sigma \circ f=\sigma \circ g$, which means $g$ is in the pseudo-iteration semigroup of $f$. However, it is a comparatively easy matter to decide which functions in the pseudo-iteration semigroup of $f$ actually commute with $f$. The intuitive idea that the functions in the pseudo-iteration semigroup that commute with $f$ are those that move the disk in the same direction as $f$ is made precise in the following proposition.

Proposition 2.2. Let $f$ be as above with Denjoy-Wolff point a with $f^{\prime}(a) \neq 0$ and suppose $\sigma$ and $V$ are as in Theorem A. Let $g$ be in the pseudo-iteration semigroup of $f$. Then $f \circ g=g \circ f$ if and only if there is an open set $U$ in $D$ such that $g(U)$ and $g(f(U))$ are contained in $V$.

Proof. $(\Rightarrow)$ Let $K=\{z:|z| \leqslant 1 / 2\}$. Since $V$ is fundamental, there is an integer $n$ so that $g\left(f_{n}(K)\right)=f_{n}(g(K)) \subset V$. Let $U$ be the interior of $f_{n}(K)$ (nonempty since $K$ interior is nonempty) so that $g(U) \subset V$. Since $f(V) \subset V$ we also have $g(f(U))=$ $f(g(U)) \subset V$.

$(\Leftarrow)$ Since $f(V) \subset V$ and $g(U) \subset V$ we have $f(g(U)) \subset V$ as well as $g(f(U)) \subset V$. Since $g$ is in the pseudo-iteration semigroup of $f$, we have

$$
\sigma(f(g(z)))=\Phi(\psi(\sigma(z)))=\psi(\Phi(\sigma(z)))=\sigma(g(f(z))) .
$$

Since $\sigma$ is one-to-one on $V$ and $f(g(z))$ and $g(f(z))$ are in $V$ whenever $z$ in in $U$, this shows $g \circ f=f \circ g$ on $U$ which implies $g \circ f=f \circ g$. 
3. The main theorem. The converse of Proposition 2.1 is almost true. We will see in $\S 4$ that the following is best possible.

THEOREM 3.1. Suppose $f$ and $g$, not automorphisms of $D$, are nonconstant analytic mappings of $D$ into $D$ that commute, and suppose the Denjoy-Wolff point of $f$ is $a$. If $\left|f^{\prime}(a)\right|<1$ then $g$ is in the pseudo-iteration semigroup of $f$. If $f^{\prime}(a)=1$, then both $f$ and $g$ are in the pseudo-iteration semigroup of $f \circ g$.

Proof. Case 1: $f^{\prime}(a)=0$. Without loss of generality, $f(z)=\alpha z^{k}+\cdots$ where $\alpha \neq 0$ and $k \geqslant 2$. Consideration of the power series for the commuting functions $f$ and $g$ shows that $g(0)=0$ also. Let $\sigma$ be as in Theorem B. For $z$ in a neighborhood of 0 , since $f(0)=g(0)=0$, we see that $\sigma \circ g \circ f$ is defined and

$$
\sigma(g(f(z)))=\sigma(f(g(z)))=[\sigma(g(z))]^{k} .
$$

That is, $\sigma \circ g$ also satisfies Böttcher's functional equation. The power series computation in part III of the proof of Theorem 1 of [5] shows that $\sigma \circ g=\lambda \sigma^{m}$, where $m$ is a positive integer and $\lambda^{k-1}=1$, which means $g$ is in the pseudo-iteration semigroup of $f$.

Case 2: $f^{\prime}(a) \neq 0$. Let $h=f \circ g$. Behan [3, p. 116] shows that since $f, g, h$ commute, they have the same Denjoy-Wolff point, $a$, and $h^{\prime}(a)=f^{\prime}(a) g^{\prime}(a)$. We claim $h^{\prime}(a) \neq 0$. If $|a|=1$, this is true because $h(D) \subset D$. If $|a|<1$ then $h^{\prime}(a)=0$ implies $g^{\prime}(a)=0$ and, by Case 1 above applied to $g, f^{\prime}(a)=0$. Since $f^{\prime}(a) \neq 0$ by hypothesis, we conclude $h^{\prime}(a) \neq 0$.

By Theorem A, we can find a fundamental set $V$ for $h$ on $D$ so that $h$ is univalent on $V$ and a domain $\Omega$ (the plane or the unit disk), a linear fractional transformation $\Phi$ mapping $\Omega$ onto $\Omega$, and $\sigma$ analytic in $D$ with $\sigma(V)$ a fundamental set for $\Phi$ on $\Omega$ such that $\sigma \circ h=\Phi \circ \sigma$.

We claim $\sigma(f(V))$ is a fundamental set for $\Phi$ on $\Omega$ also. Indeed, since $\sigma(V)$ is a fundamental set for $\Phi$ on $\Omega$, if $K$ is a compact subset of $\Omega$, there is an integer $N$ so that $\Phi_{n}(K) \subset \sigma(V)$ for $n>N$. If $n>N$, since $\sigma$ is univalent on $V$, we find that $g\left(\sigma^{-1}\left(\Phi_{n}(K)\right)\right)$ is a compact subset of $D$, where for simplicity we write $\sigma^{-1}$ for the inverse of $\sigma$ restricted to $V$. Now since $V$ is a fundamental set for $h$ on $D$, there is an integer $m$ large enough that $h_{m}\left(g\left(\sigma^{-1}\left(\Phi_{n}(K)\right)\right)\right) \subset V$. But then

$$
\begin{aligned}
\sigma(f(V)) & \supset \sigma\left(f\left(h_{m}\left(g\left(\sigma^{-1}\left(\Phi_{n}(K)\right)\right)\right)\right)\right) \\
& =\Phi_{m+1}\left(\sigma\left(\sigma^{-1}\left(\Phi_{n}(K)\right)\right)\right)=\Phi_{m+n+1}(K) .
\end{aligned}
$$

That is, if $p>N+m+1$, we have $\Phi_{p}(K) \subset \sigma(f(V))$, which means $\sigma(f(V))$ is a fundamental set for $\Phi$ on $\Omega$.

Now

$$
\sigma(f(h(z)))=\sigma(h(f(z)))=\Phi(\sigma(f(z))),
$$

which means $V, \Omega, \Phi$, and $\sigma \circ f$ also satisfy the conclusion of Theorem A for $h$. The uniqueness statement means that there is a linear fractional transformation $\varphi$ of $\Omega$ onto $\Omega$ such that $\varphi \circ \Phi \circ \varphi^{-1}=\Phi$ (that is, $\varphi$ and $\Phi$ commute) and $\sigma \circ f=\varphi \circ \sigma$. But this means $f$ is in the pseudo-iteration semigroup of $h=f \circ g$. Analogously, we 
conclude that $g$ is in the pseudo-iteration semigroup of $h$ also, say $\sigma \circ g=\psi \circ \sigma$, where $\psi$ is a linear fractional transformation mapping $\Omega$ onto $\Omega$ and commuting with $\Phi$.

For the remainder of the proof we have the hypothesis $0<\left|f^{\prime}(a)\right|<1$. If $a$ is in $D$, $V$ is a neighborhood of $a$, so there is a smaller neighborhood $\tilde{V} \subset V$ that is a fundamental set for $f$ and $f$ is univalent on $\tilde{V}$. Then $\sigma(\tilde{V})$ is a neighborhood of $\sigma(a)$ and, since $0<\left|\varphi^{\prime}(\sigma(a))\right|=\left|f^{\prime}(a)\right|<1, \sigma(\tilde{V})$ is fundamental for $\varphi$ on $\Omega$. Now $\varphi$ and $\psi$ are linear fractional transformations that commute with $\Phi$, so they commute with each other; that is, $g$ is in the pseudo-iteration semigroup of $f$.

If $|a|=1$, since $f^{\prime}(a)<1$, for any compact set $K$ in $D$, the sets $f_{n}(K)$ converge to $a$ inside a triangle with vertex at $a$ [4, Lemma 2.2, p. 74]. Moreover, since $h^{\prime}(a)<1$ and $V$ is a fundamental set for $h$, small triangles of arbitrarily large angle at $a$ are contained in $V$ [4, Proposition 3.1, p. 76]. Thus for any compact set $K$ in $D$, the set $f_{n}(K)$ is in $V$ for $n$ large enough. We may construct $\tilde{V} \subset V$ as in [4, Proposition 3.1, p. 76] so that $\tilde{V}$ is a fundamental set for $f$ on $D$ and $f$ in univalent on $\tilde{V}$.

We claim $\sigma(\tilde{V})$ is a fundamental set for $\varphi$ on $\Omega$. Since $\varphi$ and $\Phi$ commute and have $\varphi^{\prime}(\sigma(a))=f^{\prime}(a)<1$ and $\Phi^{\prime}(\sigma(a))=h^{\prime}(a)<1, \varphi$ and $\Phi$ have the same fundamental sets, in particular, $\sigma(V)$ is a fundamental set for $\varphi$ on $\Omega$. Therefore if $K$ is compact in $\Omega$, there is $n$ large enough that $\varphi_{n}(K) \subset \sigma(V)$, which means $\sigma^{-1}\left(\varphi_{n}(K)\right)$ is a compact subset of $V$ (here again we write $\sigma^{-1}$ for the inverse $\sigma$ restricted to $V$ ). Since $\tilde{V}$ is a fundamental set for $f$, there is an integer $m$ so that $f_{m}\left(\sigma^{-1}\left(\varphi_{n}(K)\right)\right) \subset \tilde{V}$. This means

$$
\sigma(\tilde{V}) \supset \sigma\left(f_{m}\left(\sigma^{-1}\left(\varphi_{n}(K)\right)\right)\right)=\varphi_{m+n}(K),
$$

which says $\sigma(\tilde{V})$ is a fundamental set for $\varphi$ on $\Omega$. Again since the linear fractional transformations $\varphi$ and $\psi$ both commute with $\Phi$, they commute with each other, and $g$ is in the local iteration semigroup of $f$.

The difficulty in the case $f^{\prime}(a)=1$ occurs in the transition from a fundamental set for $\Phi$ on $\Omega$ to a fundamental set for $\varphi$ on $\Omega$. It will be clear from the examples of the next section that this is not merely an artifact of the proof.

The following corollary strengthens the tie with Proposition 2.1.

COROllary 3.2. If $0<\left|f^{\prime}(a)\right|<1$ and $f$ commutes with $f_{n} \circ g$ for some positive integer $n$, then $g$ is in the pseudo-iteration semigroup of $f$.

Proof. From the above theorems, there is a linear fractional transformation $\tilde{\psi}$ that commutes with $\Phi$ such that $\sigma \circ f_{n} \circ g=\tilde{\psi} \circ \sigma$. This means $\Phi_{n} \circ \sigma \circ g=\tilde{\psi} \circ \sigma$ so that $\sigma \circ g=\psi \circ \sigma$ where $\psi=\Phi_{n}^{-1} \circ \tilde{\psi}$.

4. Consequences, examples, and conjectures. While in practical examples, Theorem 3.1 does not usually allow one to write down all functions $g$ that commute with $f$, the theorem does permit qualitative conclusions to be drawn about them. In this section we prove several corollaries and give examples to show the scope of the conclusions. The examples reinforce the feeling that the nature of the set of functions commuting with a given function is inextricably linked to the nature of the intertwining function $\sigma$. As in previous sections the functions $f, g$, etc. are nonconstant analytic mappings of $D$ into $D$, not automorphisms of $D$. The Denjoy-Wolff point of $f$ will be called $a$. 
Behan [3, Theorem 6, p. 116] and Shields [16, Theorem 1, p. 706] proved that commuting functions have the same Denjoy-Wolff point. The following relates the values of their derivatives there.

COROLlaRY 4.1. Let $f$ and $g$, as above, commute.

(1) If $f^{\prime}(a)=0$ then $g^{\prime}(a)=0$.

(2) If $0<\left|f^{\prime}(a)\right|<1$ then $0<\left|g^{\prime}(a)\right|<1$.

(3) If $f^{\prime}(a)=1$ then $g^{\prime}(a)=1$.

Proof. (1) If $f^{\prime}(a)=0$, without loss of generality $f(z)=\alpha z^{k}+\cdots$, where $\alpha \neq 0$ and $k \geqslant 2$. We have seen that this means $g(z)=\sigma^{-1}\left(\lambda[\sigma(z)]^{m}\right)$ in a small disk with center zero, where $\sigma$ is a solution of Böttcher's equation for $f$ univalent near zero. Now $m=0$ and $m=1$ are excluded because $g$ is nonconstant and not an automorphism of $D$ and $m \geqslant 2$ implies $g^{\prime}(0)=0$.

(2) If $0<\left|f^{\prime}(a)\right|<1$ we have seen that the data $V$, $\sigma$, and $\Omega$ of Theorem A applied to $f$ and $g$ are the same and that the linear fractional transformations $\varphi$ and $\psi$ associated with $f$ and $g$, respectively, commute. Since $\left|\varphi^{\prime}(\sigma(a))\right|=\left|f^{\prime}(a)\right|<1$, the linear fractional transformation $\varphi$ has two fixed points in the sphere, which means $\psi$ does also. Since $\sigma(V)$ is a fundamental set for $\psi$ on $\Omega$, we find $0<\left|\psi^{\prime}(\sigma(a))\right|<1$, which is our conclusion (since $g^{\prime}(a)=\psi^{\prime}(\sigma(a))$ ).

(3) If $g^{\prime}(a) \neq 1$ then $\left|g^{\prime}(a)\right|<1$ because $a$ is the Denjoy-Wolff point of $g$. Applying (1) or (2) to $g$ we conclude $f^{\prime}(a) \neq 1$.

COROLlaRY 4.2. Let $f, g_{1}, g_{2}$ be as above and suppose $g_{1}$ and $g_{2}$ commute with $f$. If $0<\left|f^{\prime}(a)\right|<1$ or if $f(z)=\alpha z^{2}+\cdots$, where $\alpha \neq 0$, then $g_{1}$ and $g_{2}$ commute with each other.

Proof. If $0<\left|f^{\prime}(a)\right|<1$, we have seen $f=\sigma^{-1} \circ \varphi \circ \sigma, g_{1}=\sigma^{-1} \circ \psi_{1} \circ \sigma$, and $g_{2}=\sigma^{-1} \circ \psi_{2} \circ \sigma$ on a set $V$ fundamental for each of $f, g_{1}$, and $g_{2}$, where $\varphi, \psi_{1}$, and $\psi_{2}$ are linear fractional transformations such that $\psi_{1}$ and $\psi_{2}$ each commute with $\varphi$. But this means $\psi_{1}$ and $\psi_{2}$ commute with each other, which implies $g_{1}$ and $g_{2}$ commute. If $f(z)=\alpha z^{2}+\cdots$, we have seen that $f(z)=\sigma^{-1}\left([\sigma(z)]^{2}\right), g_{1}(z)=$ $\sigma^{-1}\left([\sigma(z)]^{m_{1}}\right)$, and $g_{2}(z)=\sigma^{-1}\left([\sigma(z)]^{m_{2}}\right)$ in some disk with center zero (since $k=2$, the case $\lambda=1$ is the only possibility). It is easy to verify that $g_{1}$ and $g_{2}$ commute.

A trivial example shows the conclusion need not hold if $f(z)=\alpha z^{k}+\cdots$, where $k>2$, and a more interesting example shows it need not hold if $f^{\prime}(a)=1$.

EXAmple 4.3. Let $f(z)=z^{3}, g_{1}(z)=z^{4}$ and $g_{2}(z)=-z^{5}$. It is easily verified that $g_{1}$ and $g_{2}$ each commute with $f$ but not with each other.

EXAMPLE 4.4. Let $H=\{z: \operatorname{Im} z>0\}$. Let $\Delta=\{z: \operatorname{Im} z>1\} \cup\{z: \operatorname{Re} z>0$ and $0<\operatorname{Im} z \leqslant 1\}$. Let $\tilde{H}=H \backslash\{n+r i: n$ an integer, $0<r \leqslant 1\}$. Let $\pi$ be the Riemann map of $H$ onto $\tilde{H}$ so that $\pi(0)=i, \pi(1)=1+i$ and $\pi(\infty)=\infty$. The uniqueness of the Riemann mapping shows that $\pi(z+1)=\pi(z)+1$. Let $x_{1}$ and $x_{2}$ be the points such that $\pi\left(x_{1}\right)=\pi\left(x_{2}\right)=1$, named so that $x_{1}<1<x_{2}$. Let $\sigma$ be a Riemann mapping of $D$ onto $\Delta$ such that $\sigma(1)=\infty$, and let $\tilde{\sigma}=\pi \circ \sigma$. 
Now let

$$
\begin{gathered}
f(z)=\sigma^{-1}(\sigma(z)+1)=\tilde{\sigma}^{-1}(\tilde{\sigma}(z)+1), \\
g_{1}(z)=\sigma^{-1}(\sigma(z)+\delta), \quad \text { where } \delta=x_{2}-x_{1}
\end{gathered}
$$

and

$$
g_{2}(z)=\tilde{\sigma}^{-1}(\tilde{\sigma}(z)+M i)
$$

where $M$ is a positive number large enough that $g_{2}$ is defined on all of $D$, that is, $M>\sup \{\operatorname{Im} \pi(s+i): 0 \leqslant s \leqslant 1\}$. It follows from [4, §3] that 1 is the Denjoy-Wolff point of $f, g_{1}$ and $g_{2}$, and $f^{\prime}(1)=g_{1}^{\prime}(1)=g_{2}^{\prime}(1)=1$. Clearly, $g_{1}$ and $g_{2}$ each commute with $f$. To see that $g_{1}$ and $g_{2}$ do not commute with each other, let $z_{1}=\sigma^{-1}\left(x_{1}\right)$ and $z_{2}=\sigma^{-1}\left(x_{2}\right)$. Now

$$
g_{1}\left(z_{1}\right)=\sigma^{-1}\left(x_{1}+\delta\right)=\sigma^{-1}\left(x_{2}\right)=z_{2},
$$

so

$$
g_{2}\left(g_{1}\left(z_{1}\right)\right)=g_{2}\left(z_{2}\right)=\tilde{\sigma}^{-1}(1+M i)=g_{2}\left(z_{1}\right)
$$

but $g_{1}\left(g_{2}\left(z_{1}\right)\right) \neq g_{2}\left(z_{1}\right)$ because 1 is the only fixed point of $g_{1}$ in $\bar{D}$.

DEFINITION. If $h$ is a bounded analytic function on $D$, let

$$
\|h\|=\sup \{|h(z)|: z \in D\} \text {. }
$$

COROllary 4.5. If $f$, as above, has $\|f\|<1$ then there are infinitely many functions besides the natural iterates of $f$ that commute with $f$.

Proof. The hypothesis $\|f\|<1$ implies $a$ is in $D$. If $f^{\prime}(a)=0$ and $f(z)=\alpha z^{k}$ $+\cdots$, where $\alpha \neq 0$ and $k \geqslant 2$, let $\sigma$ be a solution of $\sigma \circ f=\sigma^{k}$ that is analytic and univalent in a neighborhood of zero. By Corollary 3 of [5] there is an integer $m$ so that $\tau=\sigma^{m}$ has a single-valued extension to all of $D$. Now the identity $\tau \circ f=\tau^{k}$ holds and, by Schwarz's lemma, we find that for any $\zeta$ in $\partial D$,

$$
\limsup _{r \rightarrow 1^{-}}|\tau(r \zeta)|^{k}=\limsup _{r \rightarrow 1^{-}}|\tau(f(r \zeta))| \leqslant\|f\|,
$$

so that $\|\tau\|<1$. This means there is an $N$ so that for $n>N$, the disk on which $\sigma^{-1}$ is univalent contains the set $\tau^{n}(D)$, so that the function

$$
g(z)=\sigma^{-1}\left([\tau(z)]^{n}\right)=\sigma^{-1}\left([\sigma(z)]^{m n}\right)
$$

is defined on all of $D$ and commutes with $f$. The natural iterates of $f$ have the form $f_{j}(z)=\sigma^{-1}\left([\sigma(z)]^{k^{j}}\right)$ so there are infinitely many functions $g$ of the above form that are not natural iterates of $f$.

If $f^{\prime}(a) \neq 0$, then by Theorem 3.3 of $[4, \mathrm{p} .81]$ we may, in Theorem A, taken $\Omega=\mathbf{C}$ and $\Phi(z)=s z$, where $s=f^{\prime}(a)$. The functional equation $\sigma \circ f=s \sigma$ implies

$$
\|\sigma\| \leqslant|s|^{-1} \sup \{|\sigma(z)|:|z|<\|f\|\}<\infty \text {. }
$$

Since $\sigma$ is bounded and $\sigma(V)$ is a neighborhood of zero, there is $\varepsilon>0$ so that if $|t|<\varepsilon$ then $t \sigma(D) \subset \sigma(V)$. Since $\sigma$ is univalent on $V$, if $|t|<\varepsilon$, the function $g(z)=\sigma^{-1}(t \sigma(z))$ is defined on all of $D$ and commutes with $f$. Since the natural 
iterates of $f$ have the form $f_{j}(z)=\sigma^{-1}\left(s^{j} \sigma(z)\right)$, there are infinitely many $g$ of the above form that are not natural iterates of $f$.

EXAMPLE 4.6. If $\|f\|=1$ it is possible that the only functions that commute with $f$ are its natural iterates. Let

$$
\Delta=D \cup\{z: \operatorname{Im} z>0\} \backslash\left\{2^{n}+s i: 0 \leqslant s \leqslant 1 ; n=0,1,2, \ldots\right\} .
$$

Let $\sigma$ be a Riemann map of $D$ onto $\Delta$ with $\sigma(0)=0$ and $\sigma(1)=\infty$, and let $f(z)=\sigma^{-1}\left(\frac{1}{2} \sigma(z)\right)$. Thus, $f$ is an analytic map of $D$ into $D$ with Denjoy-Wolff point $0, f^{\prime}(0)=\frac{1}{2}$ and $f(1)=1$. Now by Theorem 3.1, a function $g$ commutes with $f$ only if $g(z)=\sigma^{-1}(t \sigma(z))$, where $|t|<1$. Clearly, such a function has an analytic extension mapping $D$ into $D$ if and only if $t \Delta \subset \Delta$, which happens if and only if $t=2^{-n}$, $n=1,2,3, \ldots$. Thus the only functions commuting with $f$ are its natural iterates.

COROLlaRY 4.7. If $f$ and $g$, as above, commute and $\|f\|<1$ then for some integer $n$, $\left\|g_{n}\right\|<1$.

Proof. In the proof of Corollary 4.5, we saw that $\|f\|<1$ implies $\left\|\sigma^{m}\right\|<1$ when $f^{\prime}(a)=0$ and $\|\sigma\|<\infty$ when $f^{\prime}(a) \neq 0$. These inequalities show that for any $\delta>0$, there is $n$ large enough that $\left\|\sigma \circ g_{n}\right\|<\delta$. This means that given any neighborhood $U$ of $a$, there is $n$ large enough that $g_{n}(D) \subset U$. In particular, for $n$ large enough, $\left\|g_{n}\right\|<\frac{1}{2}(1+|a|)<1$.

EXAMPLE 4.8. It is not true that $\|f\|<1$ implies every $g$ commuting with $f$ has $\|g\|<1:$ the function

$$
f(z)=\frac{1}{4} z+\frac{1}{4} \quad\left(\text { with }\|f\|=\frac{1}{2}\right)
$$

commutes with

$$
g(z)=-\frac{1}{2} z+\frac{1}{2} \quad(\text { with }\|g\|=1) .
$$

Corollary 4.9. Suppose $f$ and $g$, as above, commute and $\left|f^{\prime}(a)\right|<1$. Then $f$ is univalent on $D$ if and only if $g$ is univalent on $D$.

Proof. If either $f$ or $g$ is univalent on $D$, since $\left|f^{\prime}(a)\right|<1$, Corollary 4.1 implies $0<\left|f^{\prime}(a)\right|<1$ and $0<\left|g^{\prime}(a)\right|<1$. We have seen that this means the data $\sigma$ and $\Omega$ of Theorem $\mathrm{A}$ are the same for $f$ and $g$. By $\S 3$ of [4], $f$ is univalent on $D$ if and only if $\sigma$ is univalent on $D$ if and only if $g$ is univalent on $D$.

EXAMPLE 4.10. It is possible, when $f^{\prime}(a)=1$, for $f$ to be univalent, but not $g$. As in Example 4.4, let $H=\{z: \operatorname{Im} z>0\}$ and

$$
\Delta=\{z: \operatorname{Im} z>1\} \cup\{z: \operatorname{Re} z>0 \text { and } 0<\operatorname{Im} z \leqslant 1\} .
$$

Let

$$
\begin{gathered}
G_{1}=\{z: \operatorname{Im} z>1\} \cup\{z: 0<\operatorname{Im} z<1 \text { and } \operatorname{Re} z \text { is not an integer }\} \\
\cup\left\{x+i: n<x<n+\frac{1}{2}, \text { some integer } n\right\},
\end{gathered}
$$

and let $G_{2}=\left\{z: z+\frac{1}{2} \in G_{1}\right\}$. Let $S$ be the Riemann surface obtained by identifying the points of $G_{1}$ and $G_{2}$ whose imaginary parts are greater than 1, and let $\nu$ be the projection of $S$ onto $G_{1} \cup G_{2}$. Then $S$ is a simply connected Riemann surface and for $\zeta$ in $S, \nu^{-1}(\nu(\zeta))$ is two points if $0<\operatorname{Im} \nu(\zeta)<1$ and $2 \operatorname{Re} \nu(\zeta)$ is not an 
integer, and one point otherwise. Let $\pi$ be the Riemann map of $H$ onto $S$ such that $\pi(\infty)=\infty, \quad \pi(0)=\lim _{\varepsilon \rightarrow 0^{+}} v^{-1}((1+\varepsilon) i)$ and $\pi(1)=\lim _{\varepsilon \rightarrow 0^{+}} \nu^{-1}(1+(1+\varepsilon) i)$.

The uniqueness of the Riemann mapping shows that $\pi(z+1)=\pi(z)+1$ (the right-hand side is the obvious abuse of notation). Let $\sigma$ be a Riemann mapping of $D$ onto $\Delta$ such that $\sigma(1)=\infty$ and let $\tilde{\sigma}=\pi \circ \sigma$. Now let $f(z)=\sigma^{-1}(\sigma(z)+1)=$ $\tilde{\sigma}^{-1}(\tilde{\sigma}(z)+1)$ and $g(z)=\tilde{\sigma}^{-1}(\tilde{\sigma}(z)+M i)$, where $M$ is a positive number large enough that $g$ is defined on all of $D$, that is, $M>\sup \{\operatorname{Im} \nu(\pi(s+i)): 0 \leqslant s \leqslant 1\}$. It follows from [4, §3] that 1 is the Denjoy-Wolff point of $f$ and $g$ and $f^{\prime}(1)=g^{\prime}(1)$ $=1$. Clearly $f$ and $g$ commute and $f$ is univalent. However, $g$ is not univalent: $\tilde{\sigma}^{-1}\left(\nu^{-1}\left(\frac{1}{4}+\frac{1}{4} i\right)\right)$ consists of two points whose images under $g$ are the same.

Shields [16] and Behan [3] show that if $f$ and $g$ commute then they have a common fixed point, namely, their Denjoy-Wolff point. If $f$ has other fixed points, must they also be shared by $g$ ? The general answer is no as we shall see in Examples 4.12 and 4.13. On the other hand, since $f$ and $g$ are so closely related, it seems likely that only a few possibilities could occur. Theorem 5.2 of $[4$, p. 92] shows that the fixed point sets of members of a continuous semigroup are the same. Since, under certain conditions, this is the set of all functions commuting with a given function, this is a result of the type we are considering. There is considerable difficulty, though, in going from the continuous semigroup case to the discrete semigroup case. The following weak result concerning the case when $f$ has exactly two fixed points can be proved.

Corollary 4.11. Suppose $0<\left|f^{\prime}(a)\right|<1, f^{\prime}$ is continuous on $\bar{D}$, and $b$ is a fixed point of $f$ on $\partial D$ such that $\overline{f(D)} \backslash\{a, b\} \subset D$. If $g$ commutes with $f$, then $b$ is a fixed point of $g$ also.

Proof. In Theorem A we may take $\sigma(a)=0, \Phi(z)=f^{\prime}(a) z$ and $\Omega=\mathbf{C}$ or $\Omega=\{z: \operatorname{Re} z>0\}$ according as $|a|<1$ or $|a|=1$ [4, §3]. By Theorem 3.1,

$$
\sigma(g(z))=g^{\prime}(a) \sigma(z) \text { and } \sigma(f(g(z)))=f^{\prime}(a) g^{\prime}(a) \sigma(z) .
$$

By Theorem 3.8 [4, p. 87], $\lim _{r \rightarrow 1^{-}} \sigma(r b)=\infty$ so $\lim _{r \rightarrow 1^{-}} \sigma(f(g(r b)))=\infty$. Now if $\lim _{r \rightarrow 1^{-}} g(r b) \neq b$ there is $\beta \neq b$ and a sequence $v_{j} \rightarrow 1$ so that $\lim _{j \rightarrow \infty} g\left(r_{j} b\right)=\beta$. This would mean

$$
\lim _{j \rightarrow \infty} f\left(g\left(r_{j} b\right)\right)=f(\beta) \in D \cup\{a\},
$$

so $\lim _{j \rightarrow \infty} \sigma\left(f\left(g\left(r_{j} b\right)\right)\right) \neq \infty$. Thus $\lim _{r \rightarrow 1^{-}} g(r b)=b$, and $b$ is a fixed point of $g$.

EXAMPLE 4.12. The conclusion of Corollary 4.11 may be false if we allow $f^{\prime}(a)=1$. Let

$$
\begin{array}{r}
G=\{x+i y: 0<x<\pi \text { or } 5 \pi / 2 \leqslant x<3 \pi \text { and } y \leqslant \cot x\} \\
\cup\{x+i y: 2 \pi<x \leqslant 5 \pi / 2 \text { and } y \leqslant \tan (x-\pi / 2)\}
\end{array}
$$

and let $\Delta=\{z: \operatorname{Re} z>0$ and $z \notin G\}$. Let $\sigma$ be a Riemann map of $D$ onto $\Delta$ with

$$
\lim _{x \rightarrow \infty} \sigma^{-1}(x)=1 \text { and } \lim _{y \rightarrow-\infty} g^{-1}\left(\frac{3 \pi}{2}+i y\right)=-1 \text {. }
$$


Let $f(z)=\sigma^{-1}(\sigma(z)+i)$ and $g(z)=\sigma^{-1}(\sigma(z)+2 \pi)$. Then 1 is the Denjoy-Wolff point of $f$ and $g, f^{\prime}(1)=g^{\prime}(1)=1$, and -1 is a fixed point of $f$ as in the hypothesis of Corollary 4.11. On the other hand, even though $f$ and $g$ commute, 1 is the only fixed point of $g$ in $\bar{D}$.

EXAMPLE 4.13. The conclusion may also be false if we allow two fixed points on $\partial D$ and have $a$ in $D$. Let $G_{1}=\{x+i y:|y| \leqslant x-1\}, G_{2}=\left\{z:-\frac{1}{2} z \in G_{1}\right\}$, and $\Delta=\mathbf{C} \backslash\left(G_{1} \cup G_{2}\right)$. Let $\sigma$ be a Riemann map of $D$ onto $\Delta$ such that $\sigma(0)=0$, let $f(z)=\sigma^{-1}\left(\frac{1}{2} \sigma(z)\right)$ and $g(z)=\sigma^{-1}\left(-\frac{1}{2} \sigma(z)\right)$. Then 0 is the Denjoy-Wolff point of $f$ and $g$, and $\sigma^{-1}(i \infty)$ and $\sigma^{-1}(-i \infty)$ are fixed points of $f$ as in the hypothesis of Corollary 4.11. On the other hand, even though $f$ and $g$ commute, 0 is the only fixed point of $g$. (It might be noted that $g \circ g$ has the same fixed points as $f$.)

These examples and geometric intuition suggest several conjectures concerning fixed points of commuting functions.

CONJECTURE 1. If $|a|=1$ and $f^{\prime}(a)<1$ then every $g$ that commutes with $f$ has the same fixed point set as $f$. (This is the discrete analogue of the continuous semigroup theorem in this case.)

CONJECTURE 2. If $f^{\prime}(a) \neq 0$, and $f$ and $g$ are commuting functions with two fixed points in common, then the fixed point sets of $f$ and $g$ are the same.

CONJECTURE 3. If $|a|<1$ and $\left|f^{\prime}(a)\right| \neq 0$ then there is an integer $n$ such that for every $g$ that commutes with $f$, the fixed point sets of $f$ and $g_{n}$ are the same.

\section{REFERENCES}

1. I. N. Baker, Zusamensetzungen ganzer Funktionen, Math. Z. 69 (1958), 121-163.

2. __ Permutable power series and regular iteration, J. Austral. Math. Soc. 2 (1962), 265-294.

3. D. F. Behan, Commuting analytic functions without fixed points, Proc. Amer. Math. Soc. 37 (1973), 114-120.

4. C. C. Cowen, Iteration and the solution of functional equations for functions analytic in the unit disk, Trans. Amer. Math. Soc. 265 (1981), 69-95.

5. Analytic solutions of Böttcher's functional equation in the unit disk, Aequationes Math. 24 (1982), 187-194.

6. A. Denjoy, Sur l'iteration des fonctions analytiques, C. R. Acad. Sci. Paris 182 (1926), 255-257.

7. P. Fatou, Sur les fonctions qui admettent plusieurs théorèmes de multiplication, C. R. Acad. Sci. Paris 173 (1921), 571-573.

8. F. Gross, Factorization of meromorphic functions, Math. Res. Center, Washington, D.C., 1972.

9. J. Hadamard, Two works on iteration and related questions, Bull. Amer. Math. Soc. 50 (1944), 67-75.

10. E. Jacobsthal, Über vertauschbare Polynome, Math. Z. 63 (1955), 243-276.

11. G. Julia, Mémoire sur le permutabilité des fractions rationelles, Ann. Sci. École Norm. Sup. 39 (1922), 131-215.

12. W. A. Pranger, Iteration of functions analytic on a disk, Aequationes Math. 4 (1970), 201-204.

13. J. F. Ritt, Prime and composite polynomials, Trans. Amer. Math. Soc. 23 (1922), 51-66.

14. __ Permutable rational functions, Trans. Amer. Math. Soc. 25 (1923), 399-448.

15. Equivalent rational substitutions, Trans. Amer. Math. Soc. 26 (1924), 221-229.

16. A. L. Shields, On fixed points of commuting analytic functions, Proc. Amer. Math. Soc. 15 (1964), 703-706.

17. G. Valiron, Fonctions analytiques, Presses Univ. de France, Paris, 1954.

18. J. Wolff, Sur l'iteration des fonctions, C. R. Acad. Sci. Paris 182 (1926), 42-43, 200-201.

Department of Mathematics, Purdue University, West Lafayette, Indiana 47907 